\title{
Avaliação da qualidade de vida relacionada à saúde entre usuários da Atenção Básica com um instrumento genérico: Duke Health Profile
}

\section{Evaluation of health related quality of life among users of Primary Care with a generic instrument: Duke Health Profile}

\author{
Jean Ezequiel Limongi ${ }^{1}$ (D) , Francisco Miguel Assis Jardine ${ }^{2}$ (D) \\ 1. Docente da Universidade Federal de Uberlândia (UFU), Uberlândia, MG, Brasil. 2. Graduado em Gestão em Saúde Ambiental pela Universidade Federal \\ de Uberlândia (UFU), Uberlândia, MG, Brasil
}

\section{Resumo}

Objetivo: analisar o estado de saúde de usuários da atenção básica por meio do DUKE, comparando os subgrupos de acordo as suas características sociodemográficas. Métodos: foi realizado estudo transversal entre usuários da atenção básica por meio de amostragem por conveniência. Para quantificar a qualidade de vida relacionada à saúde dos pacientes, foi utilizado o instrumento Duke Health Profile. Os escores das dez dimensões do instrumento foram comparados entre os subgrupos que compõem a amostra de acordo as suas características sociodemográficas. A consistência interna do Duke foi avaliada pelo coeficiente alfa de Cronbach. Resultados: participaram 484 pacientes. As mulheres e os idosos obtiveram escores menores nas escalas de saúde física, mental e geral e maiores naquelas de ansiedade, depressão e dor quando comparados aos homens e adultos, respectivamente. As escalas de saúde física e geral tiveram os coeficientes alfa de Cronbach mais elevados, 0,68 e 0,66, respectivamente. Conclusões: os baixos escores nas escalas de saúde e os altos escores nas escalas de disfunções de saúde apresentados por idosos e mulheres neste estudo representam, quantitativamente, a vulnerabilidade social e individual a que esses grupos estão submetidos. 0 uso do DUKE representa uma importante ferramenta que pode nortear ações de promoção da saúde bem como de outras políticas relacionadas à qualidade de vida.

Palavras-chave: Atenção Primária à Saúde. Autoavaliação Diagnóstica. Inquéritos e questionários. Qualidade de vida.

\begin{abstract}
Objective: to analyze the health status of users of primary health care through DUKE, comparing the subgroups according to their sociodemographic characteristics. Methods: A cross-sectional study was performed among primary care users through convenience sampling. The Duke Health Profile instrument was used to quantify the patient's health-related quality of life. The 10-dimension scores of the instrument were compared among the subgroups of the sample according to sociodemographic characteristics. Internal consistency was assessed by Cronbach's alpha coefficient. Results: A total of 484 individuals participated. Women and the elderly had lower scores on physical, mental and general health scales, and higher on anxiety, depression, and pain scores, when compared to men and adults, respectively. The physical and general health scales had the highest Cronbach alpha coefficients, 0.68 and 0.66 , respectively. Conclusions: The low scores on the health scales and the high scores on the scales of health dysfunctions presented by the elderly and women in this study quantitatively represent the social and individual vulnerability to which these groups are subjected. The use of DUKE represents an important tool that can guide health promotion actions as well as other policies related to quality of life.
\end{abstract}

Key words: Primary Health Care. Diagnostic Self Evaluation. Surveys and questionnaries. Quality of life.

\section{INTRODUÇÃO}

No Brasil, a Atenção Básica (AB) constitui-se a porta de entrada no Sistema Único de Saúde, sendo caracterizada por um conjunto de ações de saúde, no âmbito individual e coletivo, que abrangem a promoção e a proteção da saúde, a prevenção de agravos, o diagnóstico, o tratamento, a reabilitação e a manutenção da saúde ${ }^{1,2}$. Sendo assim, a $A B$ é o contato mais próximo que a população possui com a saúde pública, tendo esta, grande potencial de intervir nos fatores que influenciam a qualidade de vida relacionada à saúde (QVRS) ${ }^{3}$.

O conceito de QVRS pode ser definido como uma medida integrativa de bem-estar físico e emocional, nível de independência, relações sociais e relacionamento do indivíduo com o ambiente em que vive ${ }^{4}$. Diversos instrumentos foram propostos para quantificar a QVRS, ou seja, tornar sensações subjetivas em escores numéricos, passíveis de mensuração, análise e comparação ${ }^{5}$. Entre os mais utilizados, pode-se destacar o World Health Organization Quality of Life Assessment (WHOQOL), o Medical Outcomes Study 36-Item Short Form (SF-36), o 12-Item Short-Form Health Survey (SF-12) e o Duke Health Profile (DUKE) ${ }^{6}$.

O Duke Health Profile (DUKE) é um instrumento de medida que auxilia sobremaneira na caracterização de populações em relação ao estado de saúde. O DUKE é um questionário com 17 itens genéricos, autoaplicável ou respondido por entrevista. 
Esse instrumento foi validado, primariamente, em indivíduos usuários da atenção básica, porém também é utilizado em outras populações para mensurar o estado funcional de saúde de pessoas adultas e adolescentes, no período anterior de uma semana, tendo, assim, um panorama recente do quadro do paciente $\mathrm{p}^{7,8}$. O DUKE é um refinamento do questionário original Perfil de Saúde Duke-UNC, uma medida genérica de 63 itens que foi desenvolvida entre 1978 a 1981 por pesquisadores da Universidade Duke e da Escola de Saúde Pública da Universidade da Carolina do Norte $^{7}$. O questionário foi desenvolvido em língua inglesa e, posteriormente, foi traduzido e validado em outras 17 línguas, entre elas o português ${ }^{9,10}$. É bem aceito pelos respondentes pelo fato de ser curto, de fácil entendimento e com somente três opções de resposta. Além disso, proporciona uma gama de informações importantes por meio de um número de variáveis relativamente pequeno ${ }^{9}$.

O principal propósito do DUKE é medir o estado de saúde do indivíduo com vistas à intervenção médica e à promoção da saúde. Com esse instrumento, também é possível identificar mudanças no estado de saúde, a partir da comparação dos escores de saúde obtidos em dois ou mais momentos diferentes ${ }^{11}$. As mensurações coletadas por meio do DUKE são baseadas nas três dimensões preconizadas pela Organização Mundial de Saúde, a saúde física, mental e social ${ }^{7}$. Além disso, dispõe uma medida de saúde geral sumarizando essas três dimensões, uma de percepção de saúde e uma de autoestima, além de quatro medidas de disfunção de saúde (ansiedade, depressão, dor e incapacidade) ${ }^{7}$.

No Brasil, ainda não há estudos utilizando o DUKE; porém, no âmbito da atenção básica, esse instrumento pode auxiliar, sobremaneira, no intuito de nortear ações de promoção da saúde e cuidado integral à saúde dos usuários do Sistema Único de Saúde.

Vários estudos indicam que as propriedades psicométricas são aceitáveis e têm importante aplicação em estudos sobre promoção da saúde e intervenção médica. Porém, três dimensões de item único (percepção de saúde, dor e incapacidade) devem ser interpretadas com cautela ${ }^{6,7-11}$.

Este estudo teve por objetivo analisar o estado de saúde de usuários da atenção básica por meio do DUKE, comparando os subgrupos de acordo as suas características sociodemográficas.

\section{MÉTODOS}

Foi realizado um estudo quantitativo, transversal, com amostragem por conveniência, na Unidade de Atendimento Integrado (UAI) do município de Uberlândia, Minas Gerais, entre os meses de maio e junho de 2017. As UAls são unidades mistas, com atendimento ambulatorial na atenção básica e pronto atendimento funcionando 24 horas por dia, todos os dias da semana. O município conta com oito unidades desse tipo, distribuído em todo o seu território.
Os critérios de inclusão no estudo eram que os indivíduos fossem maiores que 18 anos de idade e usuários das unidades de atenção básica do município.

Para a coleta de dados, quatro pesquisadores abordavam os indivíduos nas salas de espera da unidade de saúde. Eram esclarecidos os objetivos da pesquisa e, depois do aceite na participação, era assinado o Termo de Consentimento Livre e Esclarecido. As questões eram respondidas na presença dos pesquisadores, porém estes não influenciavam nas respostas, apenas esclareciam termos que fugiam do domínio dos participantes.

O instrumento DUKE calcula 10 escalas de função e disfunção de saúde por meio de escores. Seis escalas medem a saúde funcional. São elas: saúde física, mental, social, geral, percepção de saúde e autoestima. Outras quatro escalas medem disfunções na saúde, são elas: ansiedade, depressão, dor e incapacidade. As escalas de saúde física, mental, social, percepção de saúde e a de incapacidade são independentes umas das outras, na medida em que nenhum dos seus itens é compartilhado, enquanto as outras escalas possuem itens compartilhados ${ }^{7}$.

Em cada um dos 17 itens do questionário DUKE, existem três opções de resposta do tipo Likert. Apenas uma opção de resposta era preenchida em cada item. As respostas eram baseadas nas condições do paciente na semana anterior à realização da entrevista com base a partir da data do preenchimento (quadro 1). O cálculo de cada escore foi realizado de acordo com o padronizado por (Parkerson, 2002) ${ }^{12}$. Para as escalas de saúde física, mental, social, geral, autoestima e percepção de saúde escores de 100 indicava o melhor estado de saúde e 0 indicava o pior estado. Para ansiedade, depressão, dor e incapacidade, escore de 100 indicava o pior estado de saúde e 0 indicava o melhor estado. Nos casos em que havia uma ou mais respostas em branco em uma das 11 escalas, o escore não era calculado para aquela escala em particular ${ }^{12}$.

Para a avaliação da consistência interna do DUKE, foi utilizado o coeficiente alfa de Cronbach. A consistência interna de um questionário é tanto maior quanto mais perto de 1 estiver o valor da estatística ${ }^{13,14}$.

Questionários foram codificados e os escores calculados de acordo com as instruções do manual do instrumento12. Um banco de dados específico para este estudo foi construído no programa Epi Info 7. 1. 3 (CDC, Atlanta, EUA). Nesse programa, foram programadas as fórmulas para os cálculos dos escores das 10 escalas que o questionário mede, os quais foram calculados, automaticamente, no momento da entrada dos dados. Além das variáveis "idade" e "sexo", já constantes no instrumento original, foram pesquisadas, também, as variáveis "Estado civil" e "Escolaridade". A estratificação das variáveis foi estabelecida com base na análise prévia do conjunto total de dados. As categorias em que havia número reduzido de dados $(\mathrm{N}<10)$ foram agrupadas com a conseguinte. A idade foi estabelecida em dois estratos, que são adultos (18 a 59 anos) e idosos (60 
anos ou mais). Todas as análises estatísticas posteriores foram realizadas neste programa.

Para a verificação da normalidade dos dados, foi utilizado o Teste de Lilliefors. Para as variáveis contínuas, foi utilizado o teste não paramétrico Wilcoxon-Mann-Whitney (variáveis anormais) ou Teste $t$ de Student (variáveis normais) ( $\alpha=5 \%$ ).

O estudo foi aprovado pelo Comitê de Ética em Pesquisa da Universidade Federal de Uberlândia (Parecer $\mathrm{n}$ 은 1.957.130/2017).

Quadro 1. Perfil de saúde de Duke, mostrando os escores brutos de cada resposta.

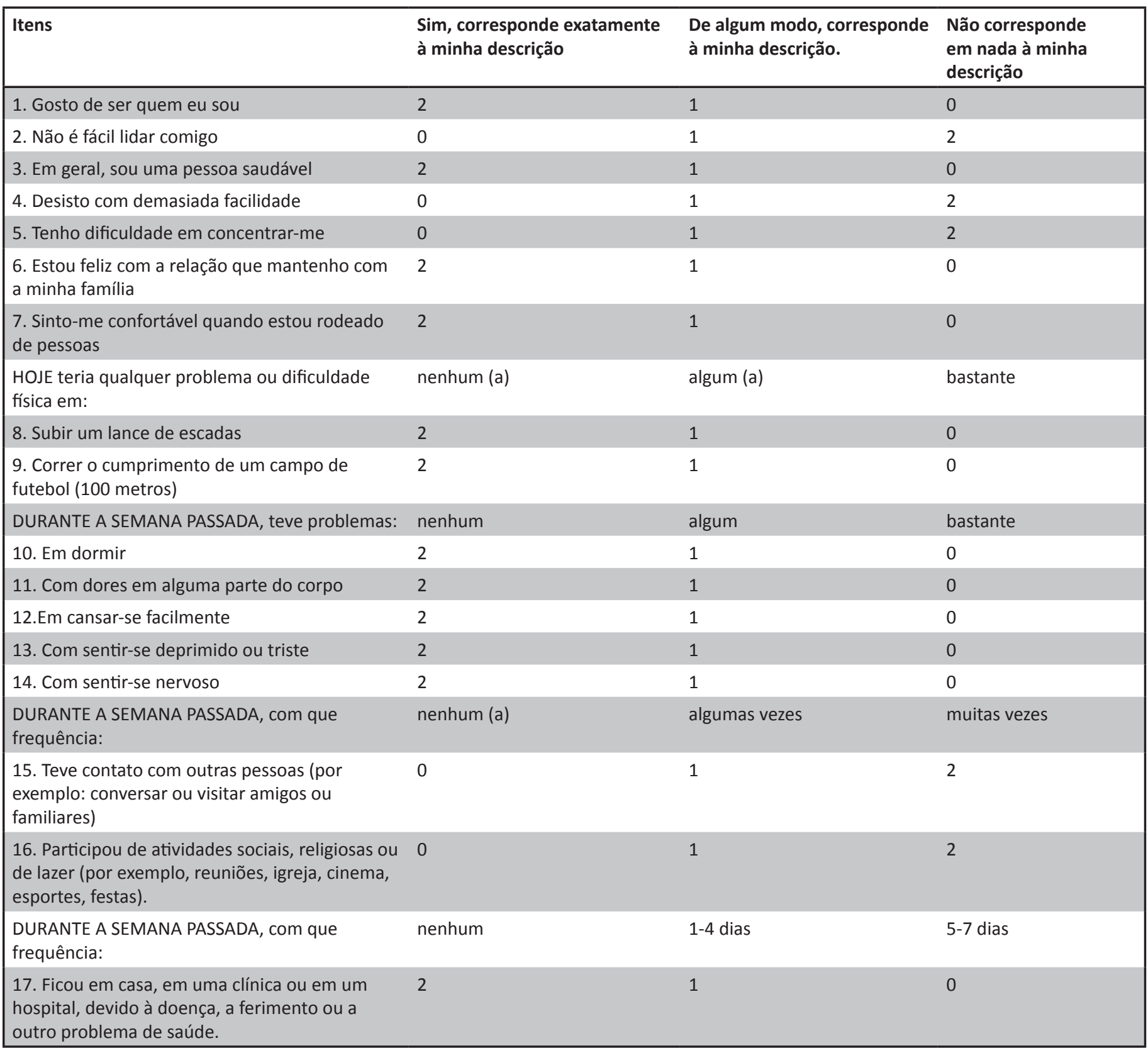

Os escores são calculados de acordo com Parkerson GR (2002)10.

\section{RESULTADOS}

Houve a participação de 484 indivíduos no estudo, sendo 252 $(52,1 \%)$ do sexo feminino. A maioria se encontrava na faixa etária adulta $(451 ; 93,2 \%)$ e com escolaridade até o ensino médio (366; 75,6\%), sendo o nível superior/pós-graduação ligeiramente mais frequente entre indivíduos do sexo feminino (64; $25,4 \%)$. Houve maior prevalência de indivíduos casados/ união estável $(251 ; 51,9)$, sendo mais comum no sexo masculino $(124 ; 53,4 \%)$ (Tabela 1$)$.

As escalas DUKE de saúde com maiores escores foram percepção de saúde $(82,23 \pm 31,97)$ e autoestima $(80,41 \pm$ $15,88)$. As escalas DUKE de disfunções de saúde com maiores 
escores foram ansiedade $(28,03 \pm 19,57)$ e depressão $(26,46 \pm$ 21,03) (Tabela 2).

As mulheres obtiveram escores menores nas escalas de saúde física, mental e geral $(p<0,0001, p=0,01, p<0,0001)$ e maiores nas escalas de ansiedade, depressão, ansiedade-depressão e dor ( $p<0,0001, p=0,01, p<0,0001, p<0,0001)$ em relação aos homens. (Tabela 3). Os idosos obtiveram escores menores nas escalas de saúde física, mental e geral $(p<0,0001, p=0,01$, $p<0,0001)$ e maiores nas escalas de ansiedade, depressão, ansiedade-depressão e $\operatorname{dor}(p<0,0001, p<0,0001, p<0,001$,

Tabela 1. Características dos participantes do estudo. p $<0,0001)$ em relação aos adultos. Indivíduos com nível superior de escolaridade obtiveram escores mais elevados na escala de saúde social $(p=0,01)$ em relação àqueles com escolaridade até ensino médio. Os indivíduos casados/união estável obtiveram escores menores na escala de saúde física $(p<0,001)$ em relação aos solteiros/viúvos/divorciados (Tabela 3).

Nenhuma das escalas teve coeficiente alfa de Cronbach acima de 0,70 , tendo as escalas de saúde física e saúde geral os melhores desempenhos, 0,68 e 0,66, respectivamente (Tabela 4).

\begin{tabular}{lcc}
\hline Variáveis & Sexo feminino & Sexo masculino \\
\hline Idade (anos) & & \\
\hline Mediana & 35,0 & 37,5 \\
\hline Mín-Máx* & $18-81$ & $18-80$ \\
\hline Escolaridade (\%) & & 76,7 \\
Até ensino médio & 74,6 & 23,3 \\
\hline Ensino Superior/ Pós-graduação & 25,4 & \\
Estado civil (\%) & & 46,6 \\
\hline Solteiro/Viúvo/Divorciado & 49,6 & 53,4 \\
\hline Casado/União estável & 50,4 & \\
\hline
\end{tabular}

* Mínimo-Máximo

Tabela 2. Escores médios para os 17 itens e as 10 escalas do Duke Health Profile (DUKE) ( $N=484$ ).

\section{Escalas de Saúde e Itens ${ }^{a}$}

Saúde Física (Soma dos itens abaixo) x 10

(8) Dificuldade de subir um lance de escadas

(9) Dificuldade de correr o cumprimento de um campo de futebol

(10) Problemas em dormir

(11) Problemas com dores em alguma parte do corpo

(12) Problemas em cansar-se facilmente

2. Saúde Mental (Soma dos itens abaixo) x 10

(1) Gosto de ser quem eu sou

(4) Desisto com demasiada facilidade

(5) Tenho dificuldade em concentrar-me

(13) Sentindo-se deprimido ou triste

(14) Sentindo-se nervoso

3. Saúde Social (Soma dos itens abaixo) x 10

(2) Não é fácil lidar comigo

(6) Estou feliz com a relação que mantenho com a minha família

(7) Sinto-me confortável quando estou rodeado de pessoas

(15) Na semana passada, teve contato com outras pessoas (por exemplo: conversar ou visitar amigos ou familiares)
Escores dos itens ${ }^{b}$

(Média \pm DP)

Escores das escalas ${ }^{b}$ (Média \pm DP) $69,97 \pm 24,10$

$72,10 \pm 35,78$

$53,40 \pm 40,74$

$77,85 \pm 34,15$

$73,86 \pm 35,59$

$72,62 \pm 34,04$

$75,70 \pm 18,24$

$93,18 \pm 20,90$

$75,10 \pm 35,61$

$67,56 \pm 36,58$

$74,38 \pm 34,57$

$67,56 \pm 36,58$

$69,93 \pm 17,66$

$59,71 \pm 40,04$

$86,98 \pm 26,64$

$86,77 \pm 25,95$

$68,28 \pm 34,22$ 
(16) ) Na semana passada, participou de atividades sociais, religiosas ou de lazer $47,41 \pm 38,04$ (por exemplo, reuniões, igreja, cinema, esportes, festas).

4. Saúde Geral (Soma das escalas de Saúde Física, Mental e Social) $\div 3$

$71,76 \pm 18,66$

5. Autopercepção de Saúde (Item abaixo) x 50

(3) Em geral, sou uma pessoa saudável

$82,23 \pm 31,92$

$82,23 \pm 31,97$

6. Autoestima (Itens 1, 2, 4, 6, 7) x 10

$80,41 \pm 15,88$

Escalas de disfunções de saúdec

7. Ansiedade (Itens 2, 5, 7, 10, 12, 14) x 8,333

$28,03 \pm 19,57$

8. Depressão (Itens 4, 5, 10, 12, 13) x 10

$26,46 \pm 21,03$

9. Dor (Item 11) x 50

$26,13 \pm 35,59$

$26,13 \pm 35,59$

10. Incapacidade (item abaixo) x 50

(17) Durante a semana passada, ficou em casa, em uma clínica ou em um hospital,

$17,35 \pm 28,02$

$17,35 \pm 28,02$ devido à doença, ferimento ou outro problema de saúde.

a Números em parênteses são os números dos itens do instrumento DUKE completo. ${ }^{b}$ Escala: 0 a 100 . Para medidas de saúde, altos escores=boa saúde; para disfunções de saúde, altos escores= saúde ruim. c Nessas escalas, os escores brutos devem ser invertidos (se 0 mude para 2 ; se 2 mude para 0; se 1 não mude. DP: desvio-padrão.

Tabela 3. Comparação das médias dos escores \pm (DP) e medianas das escalas DUKE por sexo, faixa etária, estado civil e escolaridade (análise bivariada).

\begin{tabular}{|c|c|c|c|c|c|c|c|c|}
\hline \multirow[b]{2}{*}{ Escala $^{a}$} & \multicolumn{2}{|c|}{ Sexo } & \multicolumn{2}{|c|}{ Faixa etáriab } & \multicolumn{2}{|c|}{ Estado civil } & \multicolumn{2}{|c|}{ Escolaridade } \\
\hline & $\begin{array}{r}\text { masculino } \\
(\mathrm{N}=232)\end{array}$ & $\begin{array}{r}\text { feminino } \\
(\mathrm{N}=252)\end{array}$ & $\begin{array}{r}\text { adulto } \\
(\mathrm{N}=451)\end{array}$ & $\begin{array}{r}\text { idoso } \\
(N=33)\end{array}$ & $\begin{array}{r}\text { casado/união } \\
\text { estável } \\
(\mathrm{N}=\mathbf{2 5 1})\end{array}$ & $\begin{array}{r}\text { solteiro/ } \\
\text { viúvo } \\
\text { divorciado } \\
(\mathrm{N}=\mathbf{2 3 3})\end{array}$ & $\begin{array}{r}\text { Até } \\
\text { ensino } \\
\text { médio } \\
(N=366)\end{array}$ & $\begin{array}{r}\text { ensino } \\
\text { superior/ } \\
\text { pós-graduação } \\
(\mathrm{N}=118)\end{array}$ \\
\hline Saúde física & $75,7(22,4)$ & $64,7(24,4)^{* *}$ & $71,9(22,7)$ & $43,3(26,4)^{* *}$ & $67,7(23,7)$ & $72,4(24,3)^{*}$ & $69,6(24)$ & $71,3(24,2)$ \\
\hline Saúde mental & 80 & $80 *$ & $76,3(17,9)$ & $66(20,2)^{* *}$ & $75,6(18,1)$ & $75,7(18,3)$ & $\begin{array}{r}75,3 \\
(18,3)\end{array}$ & $76,7(17,3)$ \\
\hline Saúde geral & 76,7 & $70 * *$ & $72,6(14,3)$ & $59,6(13,7)^{* *}$ & $71,3(14,5)$ & $72,2(14,7)$ & $\begin{array}{r}71,1 \\
(14,6)\end{array}$ & $73,8(14,3)$ \\
\hline Saúde social & 70 & 70 & 70 & 70 & $70,5(17,4)$ & $69,3(17,9)$ & $\begin{array}{r}68,7 \\
(17,5)\end{array}$ & $73,6(17,6)^{*}$ \\
\hline $\begin{array}{l}\text { Autopercepção de } \\
\text { saúde }\end{array}$ & 100 & 100 & $83,6(36,2)$ & $74,2(35,6)$ & $82,5(31,4)$ & $81,9(32,4)$ & $\begin{array}{r}82,8 \\
(37,5)\end{array}$ & $83,5(32,1)$ \\
\hline Autoestima & 80 & 80 & $80,9(18,4)$ & $79,1(15,1)$ & $80,5(16,3)$ & $80,3(15,4)$ & $\begin{array}{r}80,4 \\
(18,7)\end{array}$ & $81,9(16,5)$ \\
\hline Ansiedade & 25 & $25^{* *}$ & $27(19,2)$ & $41,4(20,3)^{* *}$ & $28,2(18,7)$ & $27,7(20,4)$ & $\begin{array}{r}28,3 \\
(19,8)\end{array}$ & $27,2(18,8)$ \\
\hline Depressão & 20 & $30 *$ & $25,2(20,3)$ & $43(23,6)^{* *}$ & $26,1(20,3)$ & $26,9(21,7)$ & $\begin{array}{r}27,2 \\
(21,1) \\
\end{array}$ & $24,1(20,6)$ \\
\hline $\begin{array}{l}\text { Ansiedade- } \\
\text { depressão }\end{array}$ & 21,4 & $28,6^{* *}$ & $24,6(18,3)$ & $38,1(21,1)^{* *}$ & $25,5(19,1)$ & $25,6(19,7)$ & $\begin{array}{r}25,8 \\
(19,1)\end{array}$ & $24,4(18,3)$ \\
\hline Dor & 0 & $0 * *$ & $24,1(34,5)$ & $53(39,4)^{* *}$ & $27,3(35,2)$ & $24,8(35,9)$ & $\begin{array}{r}27,5 \\
(35,9)\end{array}$ & $22(34,3)$ \\
\hline Incapacidade & 0 & 0 & $17,1(28,1)$ & $19,7(27,7)$ & $17,3(28,4)$ & $17,4(27,6)$ & $\begin{array}{r}18,3 \\
(28,5)\end{array}$ & $14,4(26,2)$ \\
\hline
\end{tabular}

Para variáveis normais, foi calculado o teste t de Student; Para variáveis não normais, foi calculado o teste de Mann-Whitney, e, neste caso, não foi apresentado o desvio-padrão.

${ }^{\mathrm{b} A d u l t o:} 18$ a 59 anos; idoso: 60 anos ou mais. ${ }^{*}$ Diferença significante entre os grupos, $\mathrm{p}<0,01$; ${ }^{*}$ Diferença significante entre os grupos, $\mathrm{p}<0,001$. DP: desviopadrão. 
Tabela 4. Coeficiente alfa de Cronbach das escalas DUKE do presente estudo, com duas interpretações sobre os valores.

\begin{tabular}{lrccc}
\hline Escala & No de itens & $\begin{array}{r}\text { Alfa de Cronbach } \\
(\mathbf{N}=484)\end{array}$ & $\begin{array}{c}\text { Interpretação do Alfa de } \\
\text { Cronbach do presente } \\
\text { estudo segundo }^{13}\end{array}$ & $\begin{array}{c}\text { Interpretação do Alfa de } \\
\text { Cronbach do presente } \\
\text { estudo segundo }^{\mathbf{1 4}}\end{array}$ \\
\hline Saúde física & 5 & 0,68 & substancial & questionável \\
Saúde mental & 5 & 0,37 & razoável & inaceitável \\
Saúde geral & 15 & 0,66 & substancial & questionável \\
Saúde social & 5 & 0,34 & razoável & inaceitável \\
Auto percepção de saúde & 1 & - & - & - \\
Autoestima & 5 & 0,32 & razoável & inaceitável \\
Ansiedade & 6 & 0,54 & moderado & pobre \\
Depressão & 5 & 0,52 & moderado & pobre \\
Dor & 1 & - & - & - \\
Incapacidade & 1 & - & - & - \\
\hline
\end{tabular}

\section{DISCUSSÃO}

Analisar a condição de saúde da população atendida é uma das premissas fundamentais da $A B$. Instrumentos de mensuração simples como o Duke auxiliam, sobremaneira, nessa atribuição, sem a necessidade de grandes recursos para a sua execução.

De forma geral, a autopercepção de saúde foi a escala com maior escore entre aquelas relacionadas ao bom estado de saúde. A utilização de autopercepção é subjetiva e muito influenciada por fatores culturais e pessoais; porém, ainda assim, tem sido amplamente utilizada, dada a facilidade de acesso15. Diferente do encontrado em outros estudos, nas comparações entre os subgrupos, não houve diferença significante quando analisados sexo, faixa etária, estado civil e escolaridade ${ }^{15,16}$.

Entre as disfunções de saúde, a ansiedade apresentou o maior escore na análise geral da casuística, com maior importância entre as mulheres e os idosos. Um estudo em João Pessoa, utilizando como instrumento de coleta de dados o Self Report Questionnaire-20 (SQR-20), também encontrou maior prevalência de ansiedade nesses subgrupos ${ }^{17}$. A alta prevalência de transtornos mentais comuns na atenção básica chama a atenção para a necessidade de práticas mais efetivas de promoção de saúde mental, com monitoramento constante por meio de instrumentos de baixo custo e fácil aplicação, como o Duke, SQR-20 entre outros disponíveis ${ }^{18}$.

As mulheres apresentaram escores menores nas escalas de saúde física, mental e geral e maiores nas escalas de ansiedade, depressão e dor. Esse padrão nos escores das escalas já foi relatado em outros estudos entre usuários da atenção básica e população geral de adolescentes e foi associado a uma maior percepção feminina de sua condição de saúde e também a uma atitude pessimista em relação às dimensões analisadas ${ }^{8,10,19}$. Homens percebem menos sua saúde como ruim quando comparado às mulheres, o que afeta a utilização dos serviços de saúde por esses usuários ${ }^{20}$.
Os idosos, como também esperado, obtiveram escores menores nas escalas de saúde física, mental e geral e maiores nas escalas de ansiedade, depressão e dor. Esse resultado deve estar associado, entre outros fatores, ao maior número de morbidades e uso de medicamentos nesse grupo da população ${ }^{21}$. Porém, esses fatores não foram pesquisados neste estudo, constituindo-se em um fator importante de limitação. Novos modelos de atendimento, interdisciplinares têm sido preconizados à população idosa, como a Avaliação Geriátrica Ampla (AGA)22. Considerando que as dimensões mais utilizadas no AGA se referem ao estado funcional, saúde mental e funcionamento social, o DUKE possui excelente aplicabilidade na mensuração dessas dimensões na população idosa, auxiliando na elaboração de propostas de promoção da saúde nesse grupo. Um estudo mostrou que o desempenho nos escores das escalas do DUKE são piores de acordo com o aumento da idade dos idosos ${ }^{23}$.

Quando comparado ao Short-Form 36 em um estudo entre pacientes acima de 65 anos, o DUKE apresentou melhor taxa de conclusão e, apesar de algumas limitações psicométricas, pode ser útil a essa população, particularmente nos pacientes mais frágeis, principalmente devido ao número reduzido de itens a serem respondidos ${ }^{24}$.

O maior nível de escolaridade foi associado com escores mais elevados na escala de saúde social. De fato, desigualdades sociais, entre elas, a escolaridade, interferem na saúde social. Além disso, afetam, também, a saúde mental e física, que são, estritamente, relacionadas e interdependentes com a primeira, além de serem fundamentais para o bem-estar dos indivíduos e da sociedade de forma geral ${ }^{25}$.

Os indivíduos casados/união estável obtiveram escores menores na escala de saúde física. Estudos sobre o assunto têm revelado que, muito além do estado civil, deve-se ser 
avaliada a qualidade conjugal, pois esta última relaciona-se mais diretamente à saúde física ${ }^{26}$. Esse aspecto, porém, não foi analisado neste estudo.

Nenhuma das escalas teve coeficiente alfa de Cronbach acima de 0,70 , tendo as escalas de saúde física e saúde geral os melhores desempenhos, 0,68 e 0,66, respectivamente. Não existe consenso sobre o valor aceitável do valor de alfa de Cronbach; em geral, varia de 0,70 a 0,95, porém alguns estudos consideram valores entre 0,60 e 0,70 como satisfatórios ${ }^{27,28}$. Entretanto, nem sempre um alfa de Cronbach alto garante um alto grau de consistência interna, isso porque o alfa é afetado sobre o número de itens do teste. Pequeno número de itens por domínio, como o DUKE, pode diminuir os valores de alfa, afetando a sua consistência interna ${ }^{27}$. Limitações semelhantes foram relatadas em jovens ${ }^{29}$, pacientes com demência30 e em idosos24. Há controvérsias em relação ao uso do coeficiente de Cronbach para avaliar instrumentos de qualidade de vida. Alguns autores o consideram inadequado, visto que, para se obter valores altos do coeficiente, deve haver um número maior de itens no instrumento; porém isso pode acarretar redundância de itens altamente correlacionados ${ }^{24}$.

De fato, o DUKE é um instrumento ambicioso, que contém domínios de saúde e disfunções de saúde em combinações de apenas 17 itens, com capacidade de uso generalizado ou em ambiente clínico, breve e com informações úteis. Por outro lado, a confiabilidade de testes curtos é mais difícil de demonstrar psicometricamente. No entanto, no desenvolvimento do DUKE, forte ênfase foi dada para a validade de cada item. Os itens são de fácil entendimento e genéricos, refletindo mudanças no estado de saúde; são comumente encontrados em contextos gerais ou em ambientes de atenção básica de saúde ${ }^{7}$. Além disso, um aspecto relevante no desenvolvimento de instrumentos de medida de saúde curtos diz respeito ao fato de que cada um dos poucos itens deve contribuir de uma forma diferente e importante para a geração da informação. Sendo assim, é esperado que o coeficiente alfa de Cronbach fosse mais baixo em instrumentos de medida mais curtos em comparação com outros mais longos e homogêneos ${ }^{7}$.

Nas escalas de saúde mental e autoestima, houve a maior discrepância de valores dos coeficientes alfa de Cronbach quando comparados com os obtidos em outros estudos ${ }^{7,9}$. Porém, é importante destacar que o coeficiente alfa de Cronbach é uma propriedade dos escores de um teste aplicado em uma amostra específica. Sendo assim, é natural que esse coeficiente varie de acordo com as populações estudadas e é imperativo que ele seja calculado todas as vezes em que o teste for aplicado ${ }^{27}$.

\section{CONCLUSÃO}

Os baixos escores nas escalas de saúde e os altos escores nas escalas de disfunções de saúde apresentados por idosos e mulheres neste estudo representam, quantitativamente, a vulnerabilidade social e individual a que esses grupos estão submetidos. Os maiores escores na escala de saúde social entre indivíduos com maior escolaridade refletem a importância da educação como determinante social da saúde. O uso do DUKE representa uma importante ferramenta que pode nortear ações de promoção da saúde bem como de outras políticas relacionadas à qualidade de vida.

\section{REFERÊNCIAS}

1. Tanaka OY. Avaliação da Atenção Básica em Saúde: uma nova proposta. Saude Soc. 2011 Out-Dez; 20(4):927-934.

2. Ministério da Saúde (BR). Política Nacional de Atenção Básica. Brasília: Ministério da Saúde; 2006. (Série E. Legislação de Saúde. Série Pactos pela Saúde).

3. Ascef BDO, Haddad JPA, Álvares J, Guerra AA Junior, Costa EA, Acurcio F de A, et al. Health-related quality of life of patients of Brazilian primary health care. Rev Saude Publica. 2017 Nov; 51(supl 2): 22S. doi: http://dx.doi.org/10.11606/ s1518-8787.2017051007134.

4. The WHOQOL Group. Study protocol for the World Health Organization project to develop a Quality of Life assessment instrument (WHOQOL). Qual Life Res 1993 Apr; 2(2): 153-159.

5. Noronha DD, Martins AME, Dias DS, Silveira MF, Paula AMBD, Haikal DSA. Qualidade de vida relacionada à saúde entre adultos e fatores associados: um estudo de base populacional. Cien Saude Colet 2016; 21(2): 463-74. doi: http://dx.doi.org/10.1590/1413-81232015212.01102015.

6. McDowell I. Measuring health: a guide to rating scales and questionnaires. 3 ed. New York: Oxford University Press; 2006.

7. Parkerson GR, Broadhead WE, Tse CK. The Duke Health Profile. A 17-item measure of health and dysfunction. Med Care 1990 Nov; 28(11): 1056-72.

8. Vo TXH, Guillemin F, Deschamps JP. Psychometric Properties of the DUKE Health Profile-adolescent Version (DHP-A): A Generic Instrument for
Adolescents. Qual Life Res 2005 Dec; 14(10): 2229-34.

9. Baumann C, Erpelding ML, Perret-Guillaume C, Gautier A, Régat S, Collin JF, et al. Health-related quality of life in French adolescents and adults: norms for the DUKE Health Profile. BMC Public Health 2011 May; 11(1): 401.

10. Parkerson GR, Gehlbach SH, Wagner EH, James SA, Clapp NE, Muhlbaier LH. The Duke-UNC Health Profile: an adult health status instrument for primary care. Med Care. 1981 Aug; 19(8): 806-28.

11. Parkerson GR, Broadhead WE, Tse CK. Development of the 17 item Duke Health Profile. Fam Pract 1991; 8(4): 396-401. doi: https://doi.org/10.1093/ fampra/8.4.396

12. Parkerson GR. User's guide for Duke Health Measures. Durham: Duke University Medical Center; 2002.

13. Landis JR, Koch GG. The measurement of observer agreement for categorial data. Biometrics; 1977 Mar; 33(1): 159-74.

14. George D, Mallery P. SPSS for Windows step by step: A simple guide and reference. 4 ed. Boston: Allyn \& Bacon; 2003.

15. Agostinho MR, Oliveira MC, Pinto AEB, Balardin GU, Harzheim E. Autopercepção da saúde entre usuários da Atenção Primária em Porto Alegre, RS. R Bras Med Fam Comum. 2010; 5(17):9-15.

16. Lima-Costa MF, Firmo JO, Uchôa E. Differences in self-rated health among older adults according to socioeconomic circumstances: the Bambuí Health and 
Aging Study. Cad Saude Publica. 2005 May; 21(3):830-9.

17. Andrade FB de, Bezerra AIC, Pontes ALF, Ferreira MO Filha, Vianna RP de T, Dias MD, et al. Saúde mental na atenção básica: um estudo epidemiológico baseado no enfoque de risco. Rev Bras Enferm. 2009;62(5):675-80.

18. Lucchese R, Sousa K de, Bonfin S do P, Vera I, Santana FR. Prevalência de transtorno mental comum na atenção primária. Acta Paul Enferm. 2014; 27(3): 200-7. doi: http://dx.doi.org/10.1590/1982-0194201400035.

19. Hanh VT, Guillemin F, Dinh Cong D, Parkerson GR, Bach Thu P, Tu Quynh $P$, et al. Health related quality of life of adolescents in Vietnam: cross-cultural adaptation and validation of the Adolescent Duke Health Profile. J Adolesc 2005 Feb; 28(1): 127-46

20. Theme MM Filha, Szwarcwald CL, Souza PR Junior. Measurements of reported morbidity and interrelationships with health dimensions. Rev Saude Publica. 2008 Feb; 42(1):73-81.

21. Tavares DM dos S, Dias FA. Capacidade funcional, morbidades e qualidade de vida de idosos. Texto Contexto - enferm. 2012 Jan-Mar; 21(1):112-20. doi: http://dx.doi.org/10.1590/S0104-07072012000100013.

22. Paixão $\mathrm{CM}$, Reichenheim ME. A review of functional status evaluation instruments in the elderly. Cad Saude Publica 2005 Jan-Feb; 21(1): 7-19. doi: http://dx.doi.org/10.1590/S0102-311X2005000100002.

23. Walker V, Perret-Guillaume C, Kesse-Guyot E, Agrinier N, Hercberg S, Galan $\mathrm{P}$, et al. Effect of Multimorbidity on Health-Related Quality of Life in Adults Aged 55 Years or Older: Results from the SU.VI.MAX 2 Cohort. PLoS One. 2016 Dec; 11(12): e0169282. doi: 10.1371/journal.pone.0169282.
24. Perret-Guillaume C, Briancon S, Guillemin F, Wahl D, Empereur F, Nguyen Thi PL. Which generic health related Quality of Life questionnaire should be used in older inpatients: comparison of the Duke Health Profile and the MOS ShortForm SF-36? J Nutr Health Aging 2010 Apr; 14(4): 325-31.

25. Moraes RSM, Silva DAS, Oliveira WF, Peres MA. Social inequalities in the prevalence of common mental disorders in adults: a population-based study in Southern Brazil. Rev bras epidemiol 2017 Jan-Mar; 20(1): 43-56. doi: http:// dx.doi.org/10.1590/1980-5497201700010004.

26. Robles TF, Slatcher RB, Trombello JM, McGinn MM. Marital quality and health: A meta-analytic review. Psychol Bull 2014; 140(1): 140-87. doi: 10.1037/ a0031859.

27. Tavakol M, Dennick R. Making sense of Cronbach's alpha. Int J Med Educ 2011; 2: 53-5. doi: 10.5116/ijme.4dfb.8dfd.

28. Souza AC, Alexandre NMC, Guirardello EB. Propriedades psicométricas na avaliação de instrumentos: avaliação da confiabilidade e da validade. Epidemiol Serv Saude 2017; 26(3): 649-59. doi: http://dx.doi.org/10.5123/s167949742017000300022

29. Parkerson GR, Broadhead WE, Tse CK. Comparison of the Duke Health Profile and the MOS Short-Form in healthy young adults. Med Care $1991 \mathrm{Jul}$ 29: 679-683.

30. Novella J, Ankri J, Morrone I, et al. Evaluation of the quality of life in dementia with a generic quality of life questionnaire: the Duke Health Profile. Dement Geriatr Cogn Disord 2001 Mar-Apr; 12(2): 158-166. doi: 10.1159/000051251.

\section{Como citar este artigo/How to cite this article:}

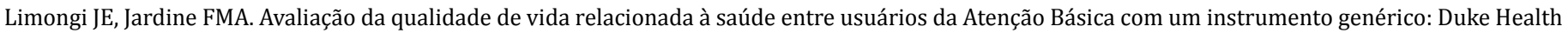
Profile. J Health Biol Sci. 2019 Jul-Set; 7(3):284-291. 